MCTP-03-27

hep-th/0305179

\title{
Hagedorn vs. Hawking-Page transition in string theory
}

\author{
Alex Buchel and Leopoldo A. Pando Zayas \\ Michigan Center for Theoretical Physics \\ Randall Laboratory of Physics, The University of Michigan \\ Ann Arbor, MI 48109-1120
}

\begin{abstract}
We study the supergravity dual to the confinement/deconfinement phase transition for the $\mathcal{N}=4 S U(N)$ SYM on $\mathbb{R} \times S^{3}$ with a chemical potential conjugate to a $U(1) \subset S O(6)_{R}$ charge. The appropriate supergravity system is a single charge black hole in $D=5 \mathcal{N}=8$ gauged supergravity. Application of the gauge/string theory holographic renormalization approach leads to new expressions for the black hole ADM mass and its generalized free energy. We comment on the relation of this phase transition to the Hagedorn transition for strings in the maximally supersymmetric plane wave background with null RR five form field strength.
\end{abstract}

May 2003 


\section{Introduction}

The gauge/string theory correspondence $[1,2]$ relates $\mathcal{N}=4 S U(N)$ supersymmetric Yang-Mills (SYM) theory to type IIB string theory on the $A d S_{5} \times S^{5}$ background. One of the most interesting aspects of this correspondence is that it allows for comparison between quantities that are not protected by symmetries. One class of these nonBPS quantities naturally arises by considering each side of the correspondence at finite temperature. For the $A d S_{5}$ in global coordinates the background geometry is dual to the SYM theory in $\mathbb{R} \times S^{3}$ which opens the possibility of phase transitions. These phase transitions are among the typical dynamical processes one expects to be able to address within the correspondence. There are various arguments supporting the existence of a confining/deconfinement phase transition for the gauge theory in the strict $N \rightarrow \infty$ limit. The nature of the transition, however, is very different at small and large 't Hooft coupling. Namely, for $\lambda \gg 1$ this confinement/deconfinement phase transition can be identified [13] with the Hawking-Page (HP) phase transition in an AdS background [14]. On the other hand, at $\lambda=0$, it was shown in [15] that the density of gauge invariant states of $\mathcal{N}=4 \mathrm{SYM}$ on $S^{3}$ exhibits a Hagedorn behavior. Based on these arguments, Polyakov [23] has suggested an interpolating formula for the density of states: $d(\Delta) \sim e^{b(\lambda) \Delta}$, with $b(\lambda) \sim$ constant for small $\lambda$ and $b(\lambda) \sim \lambda^{-1 / 4}$ for large $\lambda$.

A very peculiar behavior is expected for the free energy of the gauge theory. The $\lambda=0$ calculation [15] suggests generically a partition function near the Hagedorn transition of the form

$$
Z(x) \approx \frac{1}{\left(x_{H}-x\right) \xi^{\prime}\left(x_{H}\right)},
$$

where $x=e^{-1 / T}$. From here we see that the free energy is divergent: $Z=e^{-\beta F}$,

$$
F=\frac{1}{\beta} \ln \left(x_{H}-x\right) \xi^{\prime}\left(x_{H}\right) .
$$

On the other hand, at strong coupling we expect the free energy to be finite and given by the appropriate interpretation of the free energy of the gravity solution. The natural conjecture is that for a generic value of $\lambda$ the string theory partition function in $A d S_{5} \times S^{5}$ would have a Hagedorn temperature precisely equal to the HawkingPage critical temperature. Clearly, to substantiate this claim one needs to know the quantization of strings in $A d S_{5} \times S^{5}$ with background Ramond-Ramond fields, which 
is currently not understood ${ }^{1}$.

While quantization of strings in the full $A d S_{5} \times S^{5}$ background is not understood completely, string theory in a particular Penrose-Güven limit is exactly soluble in the light-cone [22]. The precise dictionary between string theory quantities and a particular large R-charge sector of $\mathcal{N}=4 \mathrm{SYM}$ was formulated in [3]. Moreover, the statistical mechanics of these string theories has been studied both in the canonical $[17,20]$ and the grand canonical $[18,19]$ ensembles $^{2}$. It was found, that, much like in the case of flat space, strings in plane waves have a Hagedorn temperature. In the case of the grand canonical ensemble (where in addition to the temperature one introduces a chemical potential conjugate to the $U(1) \subset S O(6)_{R}$ charge) the free energy was shown to be finite near the Hagedorn temperature [19]: $F \sim \sqrt{\beta-\beta_{H}}$. This suggests the possibility of a phase transition. However, the specific heat is negative and diverges: $c_{V} \sim\left(\beta-\beta_{H}\right)^{-3 / 2}$, obscuring the nature of the transition.

Following the relation between the Hagedorn transition and Hawking-Page phase transition outlined above, it is natural to ask whether the Hagedorn physics of strings in PP wave background is related to the physics of the Hawking-Page phase transition for the black holes in global $A d S_{5}$ that carry large $U(1)_{J} \subset S O(6)_{R}$ R-charge. The study of this connection is the main motivation of this paper.

In the next section we discuss the thermodynamics of a single charge black holes in global $A d S_{5}$ geometry. The explicit solutions were constructed previously in [4] and some aspects of the thermodynamics were studied in [5]. We present an alternative computation of the charged black hole thermodynamic properties, which utilizes the holographic renormalization approach of [6]. As we explain, this leads to a different expression for the ADM mass than the one used in, say, [5]. In section 3 we review the relevant aspects of the thermodynamics of strings in PP background in the grand canonical ensemble [18]. Unfortunately, we find that the regime where black holes have a large $J$-charge corresponds to very different values of the conjugate chemical potential $\mu_{J}$, from the one used to define the 'corresponding' PP-wave string grand canonical partition function.

\footnotetext{
${ }^{1}$ An interesting result which might help solve this problem was reported recently in [16].

${ }^{2}$ Finite temperature string theory on various pp-wave backgrounds has been also discussed in [21].
} 


\section{A single charge black holes in five dimensional gauged su- pergravity}

In this section we discuss the construction (and the thermodynamics) of a single charge black hole solution in $D=5 \mathcal{N}=8$ gauged supergravity. The asymptotic background is the global $A d S_{5}$, and the black hole would carry a $U(1) \subset S O(6)_{R}$ electric charge. This solution was originally found in [4] as a special case of the STU-model. The thermodynamic properties of these black holes were studied previously in [5]. We present a new computation for the thermodynamics, which gives different results for the ADM mass (and appropriately the Euclidean action) than the one used in $[4,5]$, and in many subsequent papers.

\subsection{The black hole geometry}

The black hole geometry can be obtained [4] as a solution of the $D=5 \mathcal{N}=8$ gauged supergravity. The relevant effective five-dimensional action is

$$
\begin{aligned}
S_{5} & =\frac{1}{4 \pi G_{5}} \int_{\mathcal{M}_{5}} d^{5} \xi \sqrt{-g} \mathcal{L} \\
& =\frac{1}{4 \pi G_{5}} \int_{\mathcal{M}_{5}} d^{5} \xi \sqrt{-g}\left(\frac{1}{4} R+\frac{1}{2} g^{2} \mathcal{V}-\frac{1}{16} H^{4 / 3} F^{2}-\frac{1}{12} H^{-2}(\partial H)^{2}\right),
\end{aligned}
$$

where $g$ is the gauge coupling, $R$ is the scalar curvature, $F_{\mu \nu}$ is a $U(1)$ field-strength tensor, and $\mathcal{V}$ is the $H$ scalar potential

$$
\mathcal{V}=2 H^{2 / 3}+4 H^{-1 / 3} .
$$

From (1) we find the following equations of motion

$$
\begin{aligned}
\square H & =H^{-1}(\partial H)^{2}+\frac{1}{2} H^{7 / 3} F^{2}-3 g^{2} H^{2} \frac{\partial \mathcal{V}}{\partial H}, \\
0 & =\partial_{\mu}\left(\sqrt{-g} H^{4 / 3} F^{\mu \nu}\right), \\
R_{\mu \nu} & =\frac{1}{2} H^{4 / 3} F_{\mu \gamma} F_{\nu}^{\gamma}+\frac{1}{3} H^{-2} \partial_{\mu} H \partial_{\nu} H-g_{\mu \nu}\left[\frac{2}{3} g^{2} \mathcal{V}+\frac{1}{12} H^{4 / 3} F^{2}\right] .
\end{aligned}
$$

We take the following ansatz for the charged black hole metric

$$
d s_{5}^{2}=-e^{-2 A / 3} f d t^{2}+e^{A / 3}\left(f^{-1} d r^{2}+r^{2}\left(d S^{3}\right)^{2}\right),
$$

where $A, f$ are functions of the radial coordinate $r$ only, and $\left(d S^{3}\right)^{2}$ is the round metric on the unit radius $S^{3}$. Additionally, we take $H \equiv H(r)$, and the only nonvanishing 
component of the gauge potential, $F=d A, A_{t} \equiv A_{t}(r)$. With this ansatz, using the equations of motion (3), we can rewrite the gravitational Lagrangian in (1) as a total derivative

$$
\sqrt{-g}\left(\frac{1}{4} R+\frac{1}{2} g^{2} \mathcal{V}-\frac{1}{16} H^{4 / 3} F^{2}-\frac{1}{12} H^{-2}(\partial H)^{2}\right)=-\left[\frac{1}{12} A^{\prime} f r^{3}+\frac{1}{2} r^{2}(f-1)\right]^{\prime},
$$

where primes denote derivatives with respect to $r$.

Omitting the computational details, the relevant two-parameter family $\{\mu, \rho\}$ of solutions of $(3)$ is

$$
e^{A}=H, \quad f=1-\frac{\mu}{r^{2}}+g^{2} r^{2} H, \quad H=1+\frac{q}{r^{2}}, \quad A_{t}=\frac{\tilde{q}}{r^{2}+q},
$$

where we introduced

$$
q=\mu \sinh ^{2} \rho, \quad \tilde{q}=\mu \sinh \rho \cosh \rho .
$$

In what follows it will be important that $\tilde{q}$ are the physical charges (i.e., the conserved charges to which Gauss's law applies). Note a useful relation

$$
\tilde{q}^{2}=q\left(q+r_{+}^{2}\right)\left(1+g^{2} r_{+}^{2}\right)
$$

\subsection{The thermodynamics}

Given the explicit single charge black solution (6), it is straightforward to extract its thermodynamics. The outer black hole horizon is at $r_{+}$, the largest non-negative zero of the function $f$,

$$
f\left(r_{+}\right)=0
$$

The inverse Hawking temperature $\beta \equiv \frac{1}{T_{H}}$, and the Bekenstein-Hawking entropy $S_{B H}$ are given by

$$
\begin{aligned}
\beta & =\frac{1}{T_{H}}=2 \pi \frac{\left(r_{+}^{2}+q\right)^{1 / 2}}{1+g^{2} q+2 g^{2} r_{+}^{2}}, \\
S_{B H} & =\frac{\mathcal{A}_{\text {horizon }}}{4 G_{5}}=\frac{\pi^{2}}{2 G_{5}} r_{+}^{2}\left(r_{+}^{2}+q\right)^{1 / 2} .
\end{aligned}
$$

The computation of the generalized free energy $\Omega \sim \frac{1}{\beta} I_{E}$ (determined from the properly regularized Euclidean gravitational action $I_{E}$ ) and the ADM mass $M$ is slightly more subtle and we discuss this in some details. In [4,5], the ADM mass is taken to be

$$
M(\mu, q) \sim \frac{3}{2} \mu+q
$$


In particular, (11) vanishes for $\mu=q=0$, which implies that the mass of global $A d S_{5}$ is assumed to be zero. But the latter statement contradicts the gauge/string theory correspondence [1,2]: it was shown in [6] that $M_{A d S_{5}}>0$, moreover its precise value exactly coincides with the (positive) dual gauge theory Casimir energy. It is instructive to see what goes wrong with the prescription for computing the ADM mass used in [4]. In [4], following the proposal of [7], the ADM mass of the geometry (4) was defined as the following surface integral at radial infinity

$$
M=-\frac{1}{8 \pi G_{5}} \int_{S^{3}} N\left(K-K_{0}\right),
$$

where $N$ is the norm of the time-like Killing vector and $K$ is the extrinsic curvature, dependent on the black hole parameters $\{\mu, q\}$; finally $K_{0}$ is taken to be the corresponding extrinsic curvature of the global $A d S_{5}$ geometry. Literally following this prescription for the single charge black of interest here does not give (11), rather we find $M \sim g^{2} q r^{2} \rightarrow \infty$. The technical reason for this is that the 5D gauge fields of the black hole solution modify the first subleading (as $r \rightarrow \infty$ ) correction of $f$ in (6), that can not be subtracted by comparing with the 'uncharged' global $A d S_{5}$ geometry. Now, the finite mass term results from the second subleading term in $f$, and thus the subtraction (12) necessarily would give diverging result ${ }^{3}$.

In the rest of this section we present the modified prescription for computing $I_{E}, M$, that, first of all, gives finite results for the above quantities. Additionally, in the limit of vanishing charge and the nonextremality parameter we recover the global $A d S$ mass of [6]. Our prescription relies on the Maldacena proposal for the existence of the dual (local) four dimensional quantum field theory for the black hole geometry (4). In a sense, this is a simple application of the renormalization ideas of [6], which was originally implemented in [9] for the non-extremal black holes in AdS, charged under the diagonal $U(1)_{\text {diag }} \subset U(1)^{3} \subset S O(6)_{R}$. Unlike the single charge black hole solutions of interest here, for the $U(1)_{\text {diag }}$ charged black holes, the ADM mass can also be computed [10] by subtracting the global $A d S_{5}$ geometry as a reference, (12). Both computations $[10,9]$ lead to the same results for the black hole mass and the regularized Euclidean gravitational action.

\footnotetext{
${ }^{3}$ The application of the procedure of [7] to the recently studied black hole solution [8] would also give diverging result for the ADM mass. Again, the problem appear to be due to the additional matter fields compared to the extremal geometry.
} 
We begin by summarizing our results:

$$
\begin{aligned}
& I_{E}=\frac{\beta \pi}{G_{5}}\left(-\frac{1}{8} \mu-\frac{1}{12} q^{2} g^{2}+\frac{3}{32} g^{-2}+\frac{1}{4} r_{+}^{2}\right), \\
& M=\frac{\pi}{G_{5}}\left(\frac{3}{8} \mu+\frac{1}{4} q-\frac{1}{12} q^{2} g^{2}+\frac{3}{32} g^{-2}\right) .
\end{aligned}
$$

Notice that using (10) and (13) we find the expected thermodynamics relation ${ }^{4}$

$$
I_{E}=\beta\left(M-\mu_{\tilde{q}} \tilde{q}\right)-S_{B H}
$$

where $\mu_{\tilde{q}}$ is the chemical potential conjugate to the physical black hole charge $\tilde{q}$, related to the gauge potential $A_{t}(6)$ at the horizon, $r=r_{+}$

$$
\mu_{\tilde{q}}=\left.\frac{\operatorname{vol}\left(S^{3}\right)}{8 \pi G_{5}} A_{t}(r)\right|_{r=r_{+}}=\frac{\pi}{4 G_{5}} \frac{\tilde{q}}{r_{+}^{2}+q} .
$$

To obtain (14) we used (8). In the limit of vanishing charge we find from (13)

$$
\left.M\right|_{q=0}=\frac{3 \pi}{32 g^{2} G_{5}}+\frac{3 \pi \mu}{8 G_{5}},
$$

which with identification $\mu \equiv r_{0}^{2}, g \equiv \frac{1}{\ell}$ is precisely the result obtained in [6] for the Schwarzschild black hole in global $A d S_{5}$.

Let us compute the renormalized (in the sense of [6]) Euclidean gravitational action $I_{E}$ of (1). First, we regularize (1) by introducing the boundary $\partial \mathcal{M}_{5}$ at fixed (large) $r$ with the unit orthonormal space-like vector $n^{\mu} \propto \delta_{r}^{\mu}$

$$
\begin{aligned}
S_{5}^{r} & =\frac{1}{4 \pi G_{5}} \int_{r_{+}}^{r} d r \int_{\partial \mathcal{M}_{5}} d^{4} \xi \sqrt{g_{E}} \mathcal{L}_{E}=-\frac{1}{4 \pi G_{5}} \int_{r_{+}}^{r} d r \int_{\partial \mathcal{M}_{5}} d^{4} \xi \sqrt{-g} \mathcal{L} \\
& =\frac{1}{4 \pi G_{5}} \int_{r_{+}}^{r} d r\left[\frac{1}{12} A^{\prime} f r^{3}+\frac{1}{2} r^{2}(f-1)\right]^{\prime} \int_{\partial \mathcal{M}_{5}} d^{4} \xi \\
& =\left.\frac{\beta \pi}{2 G_{5}}\left[\frac{1}{12} A^{\prime} f r^{3}+\frac{1}{2} r^{2}(f-1)\right]\right|_{r_{+}} ^{r},
\end{aligned}
$$

where the subscript ${ }_{E}$ represents that all the quantities are to be computed in Euclidean signature. We used (5) to obtain the second identity in (17). As usual, to have a welldefined variational problem in the presence of a boundary requires the inclusion of the Gibbons-Hawking $S_{G H}$ term

$$
\begin{aligned}
S_{G H} & =-\frac{1}{8 \pi G_{5}} \int_{\partial \mathcal{M}_{5}} d^{4} \xi \sqrt{h_{E}} \nabla_{\mu} n^{\mu} \\
& =\frac{\beta \pi}{2 G_{5}}\left[-\frac{1}{12} A^{\prime} f r^{3}-\frac{1}{4} r^{3} f^{\prime}-\frac{3}{2} r^{2} f\right],
\end{aligned}
$$

\footnotetext{
${ }^{4}$ Black holes with 'hair' for which similar relation can be proved are discussed in $[11,8]$.
} 
where $h_{\mu \nu}$ is the induced metric on $\partial \mathcal{M}_{5}$

$$
h_{\mu \nu} \equiv g_{\mu \nu}-n_{\mu} n_{\nu}
$$

Finally, as in [6], we supplement the combined regularized action $\left(S_{5}^{r}+S_{G H}\right)$ by the appropriate boundary counterterms constructed from the local ${ }^{5}$ metric invariants on the boundary $\partial \mathcal{M}_{5}$

$$
\begin{aligned}
S^{\text {counter }} & =\frac{1}{4 \pi G_{5}} \int_{\partial \mathcal{M}_{5}} d^{4} \xi\left(\alpha_{1} \sqrt{h_{E}}+\alpha_{2} R_{4} \sqrt{h_{E}}\right) \\
& =\frac{\beta \pi}{2 G_{5}}\left[\alpha_{1} e^{A / 6} f^{1 / 2} r^{3}+6 \alpha_{2} r f^{1 / 2} e^{-A / 6}\right],
\end{aligned}
$$

where $R_{4} \equiv R_{4}\left(h_{E}\right)$ is the Ricci scalar constructed from $h_{\mu \nu}$, and $\alpha_{i}$ are coefficients that can depend only on the curvature of the asymptotic $A d S_{5}$ geometry ${ }^{6}$. The counterterm parameters $\alpha_{i}$ are fixed in such a way that the renormalized Euclidean action $I_{E}$ is finite

$$
I_{E} \equiv \lim _{r \rightarrow \infty}\left(S_{5}^{r}+S_{G H}+S^{\text {counter }}\right), \quad\left|I_{E}\right|<\infty .
$$

Using the explicit solution (6) we find the answer (13) for $I_{E}$ and

$$
\alpha_{1}=\frac{3}{2} g, \quad \alpha_{2}=\frac{1}{8} g^{-1} .
$$

We now proceed to the computation of the ADM mass for the background (1). Following [6] we define

$$
M=\int_{\Sigma} d^{3} \xi \sqrt{\sigma} N_{\Sigma} \epsilon
$$

where $\Sigma \equiv S^{3}$ is a spacelike hypersurface in $\partial \mathcal{M}_{5}$ with a timelike unit normal $u^{\mu}, N_{\Sigma}$ is the norm of the timelike Killing vector in (4), $\sigma$ is the determinant of the induced metric on $\Sigma$, and $\epsilon$ is the proper energy density

$$
\epsilon=u^{\mu} u^{\nu} T_{\mu \nu}
$$

The quasilocal stress tensor $T_{\mu \nu}$ for our background is obtained from the variation of the full action

$$
S_{t o t}=S_{5}^{r}+S_{G H}+S^{\text {counter }}
$$

\footnotetext{
${ }^{5}$ The locality condition is very important and it follows from the locality of the dual gauge theory. In our case this dual gauge theory is the $\mathcal{N}=4 \mathrm{SYM}$ in the deconfined phase with a given chemical potential conjugate to the $R$-charge. Example where locality condition does not hold will be discussed elsewhere [12].

${ }^{6}$ For this reason the values of $\alpha_{i}$ must be the same as the corresponding parameters in [6].
} 
with respect to the boundary metric $\delta h_{\mu \nu}$

$$
T^{\mu \nu}=\frac{2}{\sqrt{-h}} \frac{\delta S_{t o t}}{\delta h_{\mu \nu}}
$$

Explicit computation yields

$$
T^{\mu \nu}=\frac{1}{8 \pi G_{5}}\left[-\Theta^{\mu \nu}+\Theta h^{\mu}-2 \alpha_{1} h^{\mu \nu}+4 \alpha_{2}\left(R_{4}^{\mu \nu}-\frac{1}{2} R_{4} h^{\mu \nu}\right)\right],
$$

where

$$
\Theta^{\mu \nu}=\frac{1}{2}\left(\nabla^{\mu} n^{\nu}+\nabla^{\nu} n^{\mu}\right), \quad \Theta=\operatorname{Tr} \Theta^{\mu \nu} .
$$

It is straightforward to verify that with $\alpha_{i}$ as in (22), the mass as defined in (23) is finite, and is given by (13).

\subsection{The phase diagram of a single charge black hole}

To make the connection with the dual $\mathcal{N}=4 S U(N)$ SYM theory on $\mathbb{R} \times S^{3}$ we recall $[2]$

$$
\frac{1}{g^{3} G_{5}}=\frac{2 N^{2}}{\pi}, \quad g=\ell^{-1} .
$$

We would like to identify the thermodynamics characteristics of the single charge black hole computed in the previous section $\left\{I_{E}, M, S_{B H} ; T_{H}, \mu_{\tilde{q}}\right\}$ with the appropriate gauge theory quantities $\left\{\Omega, E, S, T, \mu_{J}\right\}$

$$
\left\{I_{E}, M, S_{B H} ; T_{H}, \mu_{\tilde{q}}\right\} \longleftrightarrow\left\{\Omega, E, S ; T, \mu_{J}\right\}
$$

where the thermodynamic potential $\Omega$ is related to the Helmholtz free energy $F$ in the standard way

$$
\Omega=F-\mu_{J} J=E-T S-\mu_{J} J
$$

The identification we are after must preserve the relation (14) and satisfy the first law of thermodynamics for the grand canonical ensemble with $\left\{T, \mu_{J}\right\}$ as independent variables

$$
d \Omega=-S d T-J d \mu_{J}
$$

We propose to identify

$$
\begin{aligned}
T & \equiv T_{H}, \\
S & \equiv S_{B H}, \\
\mu_{J} & \equiv \mu_{\tilde{q}}
\end{aligned}
$$


Given (33), (14), the first law (32) uniquely determines ${ }^{7}$

$$
\begin{aligned}
& \Omega \equiv T_{H} I_{E}+\frac{g^{2} \pi}{12 G_{5}} q^{2}, \\
& E \equiv M+\frac{g^{2} \pi}{12 G_{5}} q^{2} .
\end{aligned}
$$

We do not have an independent way of justifying the identification (34), apart from the argument presented above. Note that for the vanishing physical charge $\tilde{q}, q$ vanishes as well, and we get the standard identification $\beta F \equiv I_{E}$.

In what follows we set the five dimensional gauge coupling (or equivalently the $A d S_{5}$ scale) $g=1$. Thus, from (29) all the gauge theory thermodynamics quantities would scale $\propto N^{2}$, as appropriate for the deconfined phase. On the other hand, in the confined phase we expect these thermodynamic potentials to scale $\propto N^{0}$, effectively zero in the large $N$ limit. For fixed temperature $T \equiv T_{H}$ and chemical potential $\mu_{J}$, the physical gauge theory phase ( in the large $N$ limit $^{8}$ ) has chemical potential $\Omega_{\text {phys }}$ as

$$
\frac{1}{N^{2}} \Omega_{p h y s}\left(T, \mu_{J}\right)=\min \left\{\frac{1}{N^{2}} \Omega\left(T, \mu_{J}\right), 0\right\} .
$$

We expect the confinement/deconfinement phase transition to occur at $\left\{T\left(\mu_{J}\right), \mu_{J}\right\}$ such that

$$
\Omega\left(T\left(\mu_{J}\right), \mu_{J}\right)=0
$$

We found the best way to parametrize the thermodynamics is to use the analog of the 'unphysical' charge $q$ in the black hole case ${ }^{9}$. The summary of the thermodynamics is as follows.

- The critical curves for the generalized free energy $\Omega$ as a function of $(T, q)$ are presented in Fig. 1. The green line $\left(T_{\text {green }}(q)\right)$ corresponds to the vanishing of the outer black hole horizon. For values of $(T, q)$ below it, both the $r_{+}$and the $\Omega$ are imaginary. The red line $\left(T_{\text {red }}(q)\right)$ corresponds to the confinement/deconfinement phase transition as in (36): for values of $(T, q)$ in the strip between the red and the green lines $\Omega>0$. For $(T, q)$ above the phase transition curve, $\Omega<0$. Asymptotically as $q \rightarrow+\infty$ we

\footnotetext{
${ }^{7}$ Strictly speaking there is a single overall constant, independent of temperature and the chemical potential, in the definition of $E$ and $\Omega$. The latter constant must be set to zero in order to reproduce the result for the agreement of gauge theory Casimir energy and the ADM mass [6] at $\left\{T=0, \mu_{J}=0\right\}$.

${ }^{8}$ There is no phase transition, but rather a crossover for finite $N$.

${ }^{9}$ Note that the identification (33) for the chemical potential implies that $J \equiv \tilde{q}$.
} 


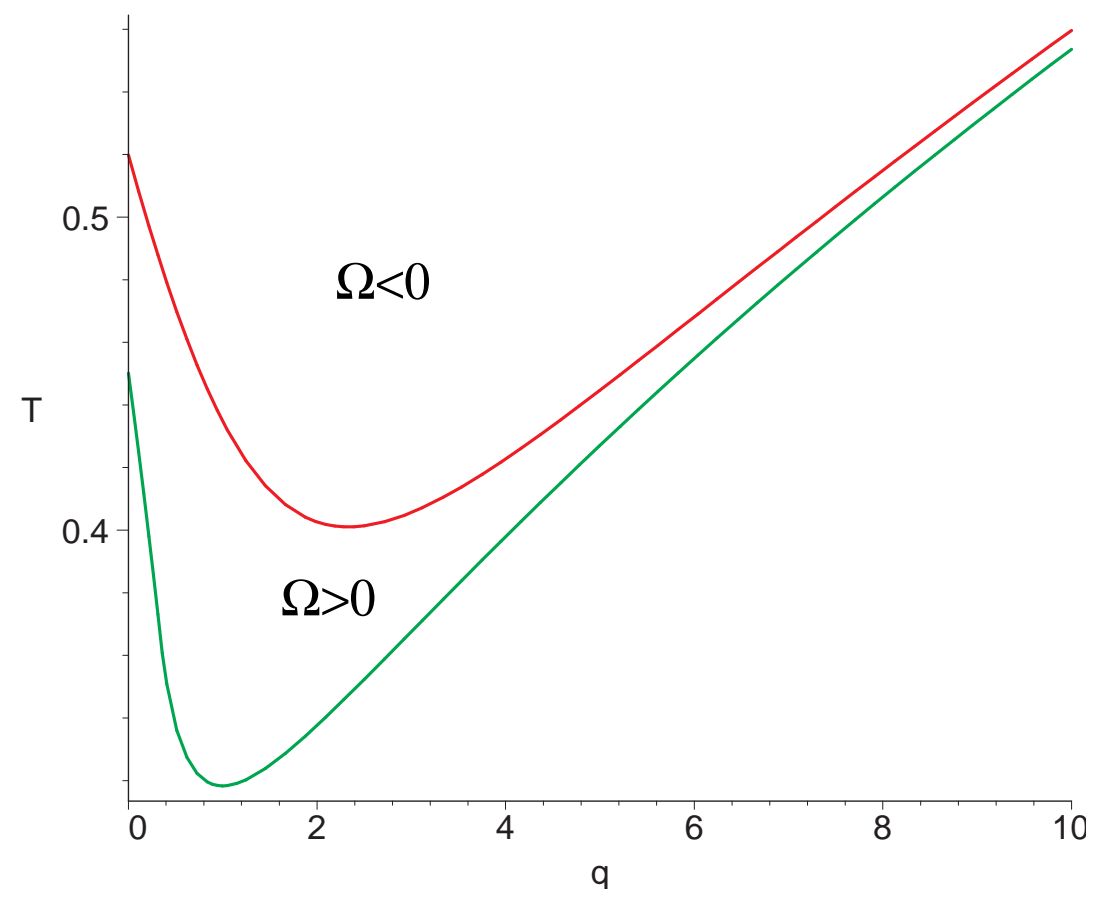

Figure 1: The critical curves for the thermodynamic potential $\Omega(T, q)$, where $q \in$ $[0,+\infty), T \in[0,+\infty)$. Below the green line $\operatorname{Im} \Omega \neq 0$. In a strip between the green and the red lines $\Omega>0$, and above the red line $\Omega<0$. The red line corresponds to the critical line for the confinement/deconfinement phase transition, (36).

have

$$
\begin{aligned}
T_{\text {green }} & =\frac{1}{2 \pi} q^{1 / 2}+\frac{1}{2 \pi} q^{-1 / 2}, \\
T_{\text {red }} & =T_{\text {green }}+\frac{9}{16 \pi} q^{-3 / 2}+O\left(q^{-5 / 2}\right) .
\end{aligned}
$$

- At the phase transition temperature $T_{\text {critical }} \equiv T_{\text {red }}$, the chemical potential $\frac{\mu_{J}}{N^{2}}$ and the charge $J$ are shown in Fig. 2 and Fig. 3 correspondingly. Asymptotically as $q \rightarrow+\infty$ we find

$$
\frac{\mu_{J}}{N^{2}}=\frac{1}{2}+\frac{3}{16} q^{-1}+O\left(q^{-2}\right)
$$

and

$$
J=q+\frac{3}{8}+O\left(q^{-1}\right)
$$

- For completeness we also present the asymptotic $q \rightarrow+\infty$ behavior of $S_{c} \equiv S\left(T_{\text {critical }}, q\right)$, 


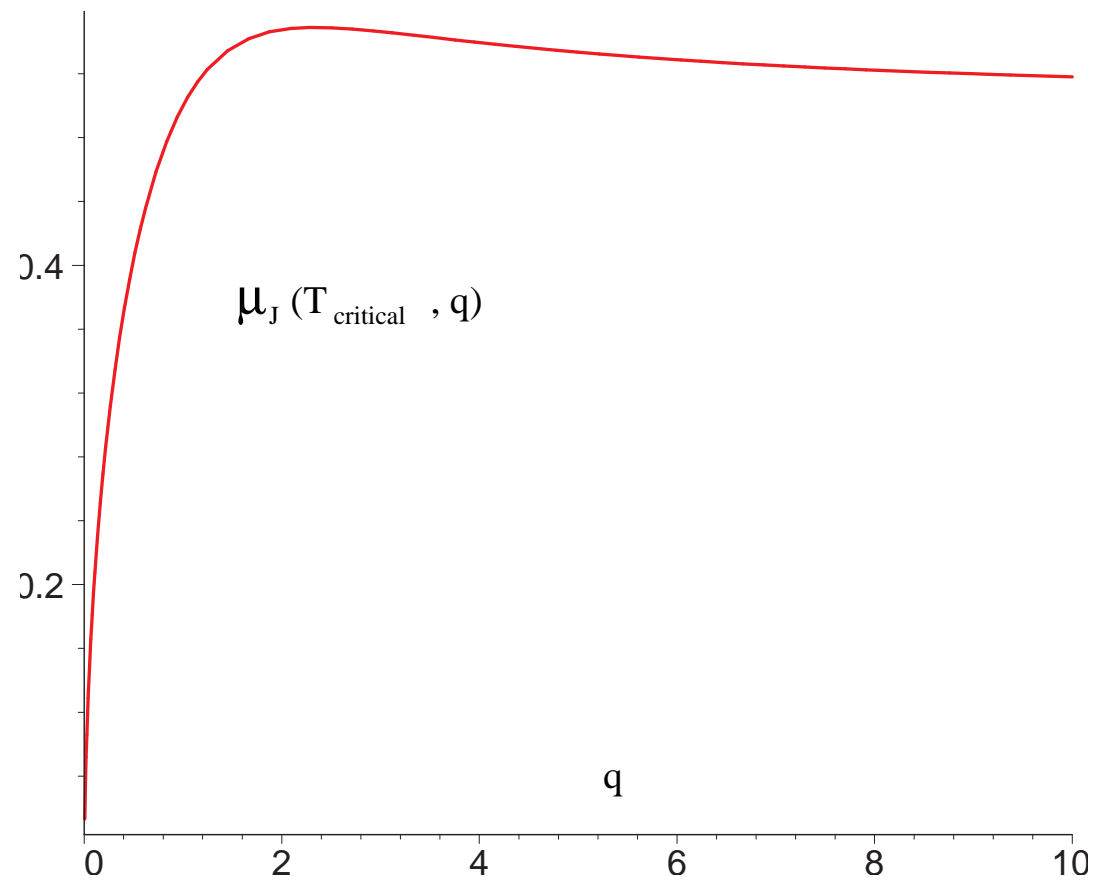

Figure 2: The chemical potential $\mu_{J}$ conjugate to the charge $J$ as a function of $q$, at the critical temperature $T=T_{\text {critical }}$.

$$
\begin{aligned}
& E_{c} \equiv E\left(T_{\text {critical }}, q\right), r_{\text {hor }} \equiv r_{+}\left(T_{\text {critical }}, q\right) \\
& \frac{1}{N^{2}} S_{c}=\frac{3 \pi}{4} q^{-1 / 2}+\frac{3 \pi}{4} q^{-3 / 2}+O\left(q^{-5 / 2}\right), \\
& \frac{1}{N^{2}} E_{c}=\frac{1}{2} q+\frac{3}{4}+O\left(q^{-1}\right), \\
& r_{\text {hor }}=\frac{\sqrt{3}}{2} q^{-1 / 2}+\frac{\sqrt{3}}{4} q^{-3 / 2}+O\left(q^{-5 / 2}\right) .
\end{aligned}
$$

\section{Plane wave background at finite temperature}

One of the most interesting aspects the plane wave background is that, being a PenroseGüven limit of $A d S_{5} \times S^{5}$, it can be used as an specific example [3] of the gauge/string theory correspondence on one side of which there is an exactly solvable theory [22].

The aspect we review in this section is the thermodynamic properties of this background and its implications for the field theory. The finite temperature partition function of string theory in the plane wave background with RR null 5-form field has been obtained in [17-20]. One of the most salient features that has been established is the existence of a Hagedorn temperature for strings in this background.

To clarify the relation between the string theory and the field theory quantities it 


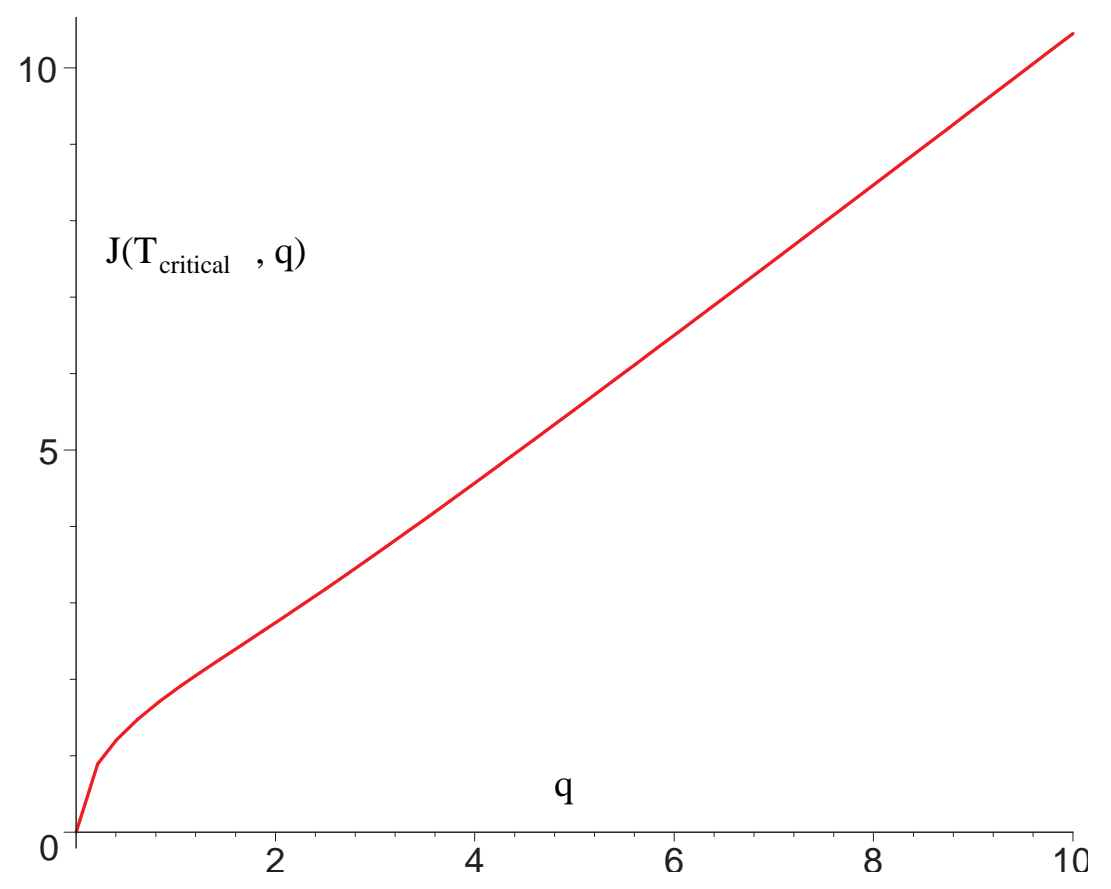

Figure 3: The $U(1) \subset S O(6)_{R}$ charge $J$ as a function of $q$, at the critical temperature $T=T_{\text {critical }}$.

is convenient to follow the Penrose limit suggested by Tseytlin and presented in [24]. Namely, we start with

$$
x^{+}=t, \quad x^{-}=R^{2}(t-\psi),
$$

where $R$ is the AdS radius and $\psi$ is a coordinate parameterizing the great circle of $S^{5}$. The Penrose-Güven limit along this null geodesic results in the standard maximally supersymmetric IIB plane wave background with null RR 5-form flux and the following relation between string and gauge quantities:

$$
\frac{2 p_{+}}{\mu}=E-J, \quad 2 \mu \alpha^{\prime} p_{-}=\frac{J}{\sqrt{\lambda}} .
$$

In the field theory interpretation $E$ is the energy of states in $\mathbb{R} \times S^{3}$ and $J$ is the $U(1)$ $\mathrm{R}$-charge of the corresponding state.

Let us, following $[18,19]$, define a slightly more general partition function of the form

$$
\mathcal{Z}(a, b ; \mu)=\operatorname{Tr}_{\mathcal{H}} e^{-b p_{-}-a p_{+}} .
$$

One of the virtues of this partition function is that it makes explicit the interpretation in the grand canonical ensemble as introducing a chemical potential. The partition 
function of an ideal gas of IIB strings in the maximally supersymmetric plane wave background can be written in term of the single string partition function for bosonic $Z_{1}^{B}$ and fermionic $Z_{1}^{F}$ modes:

$$
\ln \mathcal{Z}(a, b ; \mu)=\sum_{r=1}^{\infty} \frac{1}{r}\left[\mathcal{Z}_{1}^{B}(a r, b r ; \mu)-(-1)^{r} \mathcal{Z}_{1}^{F}(a r, b r ; \mu)\right] .
$$

The single string partition function can be written as

$$
\mathcal{Z}_{1}(a, b ; \mu)=\operatorname{Tr}_{\mathcal{H}} e^{-b p_{-}-a p_{+}} .
$$

Most of our conclusions will rely on the single string approximation. Using the expression for the light-cone Hamiltonian obtained in [22]:

$$
\begin{aligned}
H_{l c} & =\frac{1}{\alpha^{\prime} p_{-}}\left[m \sum_{i=1}^{8}\left(a_{0}^{i \dagger} a_{0}^{i}+S_{0}^{i \dagger} S_{0}^{i}\right)\right. \\
& \left.+\sum_{n=1}^{\infty} \sqrt{n^{2}+m^{2}}\left(\sum_{i=1}^{8}\left(a_{n}^{i \dagger} a_{n}^{i}+\tilde{a}_{0}^{i \dagger} \tilde{a}_{0}^{i}\right)+\left(S_{n}^{i \dagger} S_{0}^{i}+\tilde{S}_{0}^{i \dagger} \tilde{S}_{0}^{i}\right)\right)\right]
\end{aligned}
$$

where $m=\mu \alpha^{\prime} p_{-}$and identifying $\tau_{2}=a / 2 \pi \alpha^{\prime} p_{-}$we find

$$
Z_{1}(a, b ; \mu)=\frac{a V_{L}}{4 \pi^{2} \alpha^{\prime}} \int_{0}^{\infty} \frac{d \tau_{2}}{\tau_{2}^{2}} \int_{-1 / 2}^{1 / 2} d \tau_{1} e^{-\frac{a b}{2 \pi \alpha^{\prime} \tau_{2}}} z_{l c}^{(0,0)}\left(\tau, \mu a / 2 \pi \tau_{2}\right) z_{l c}^{(0,1 / 2)}\left(\tau, \mu a / 2 \pi \tau_{2}\right),
$$

where $z_{l c}^{(0,0)}$ and $z_{l c}^{(0,1 / 2)}$ are roughly the partition functions of massive two-dimensional bosons and fermions respectively and we refer the reader to [17] for the precise notation and further details. Our main concern will be with the Hagedorn temperature although other thermodynamic quantities such as the free energy can also be calculated [17-20]. We define the Hagedorn temperature as the value above which the partition function starts diverging.

$$
\frac{b}{16 \mu \alpha^{\prime}}=\gamma_{0}\left(\frac{a \mu}{2 \pi}\right)-\gamma_{1 / 2}\left(\frac{a \mu}{2 \pi}\right) .
$$

where on the right hand side figure the difference of the Casimir energies for bosons and fermions [17]. The above expression can be made explicit in terms of integrals or Bessel functions [17-20].

Let us now turn to the meaning of the expression for the Hagedorn temperature in terms of the field theory variables. Taking into account (42) and (45), the temperature and chemical potential of the gauge theory are

$$
\beta_{Y M}=\frac{1}{T_{Y M}}=\frac{a \mu}{2}, \quad \mu_{J}=\frac{b}{2 \mu \alpha^{\prime} \sqrt{\lambda}}-\frac{a \mu}{2} .
$$




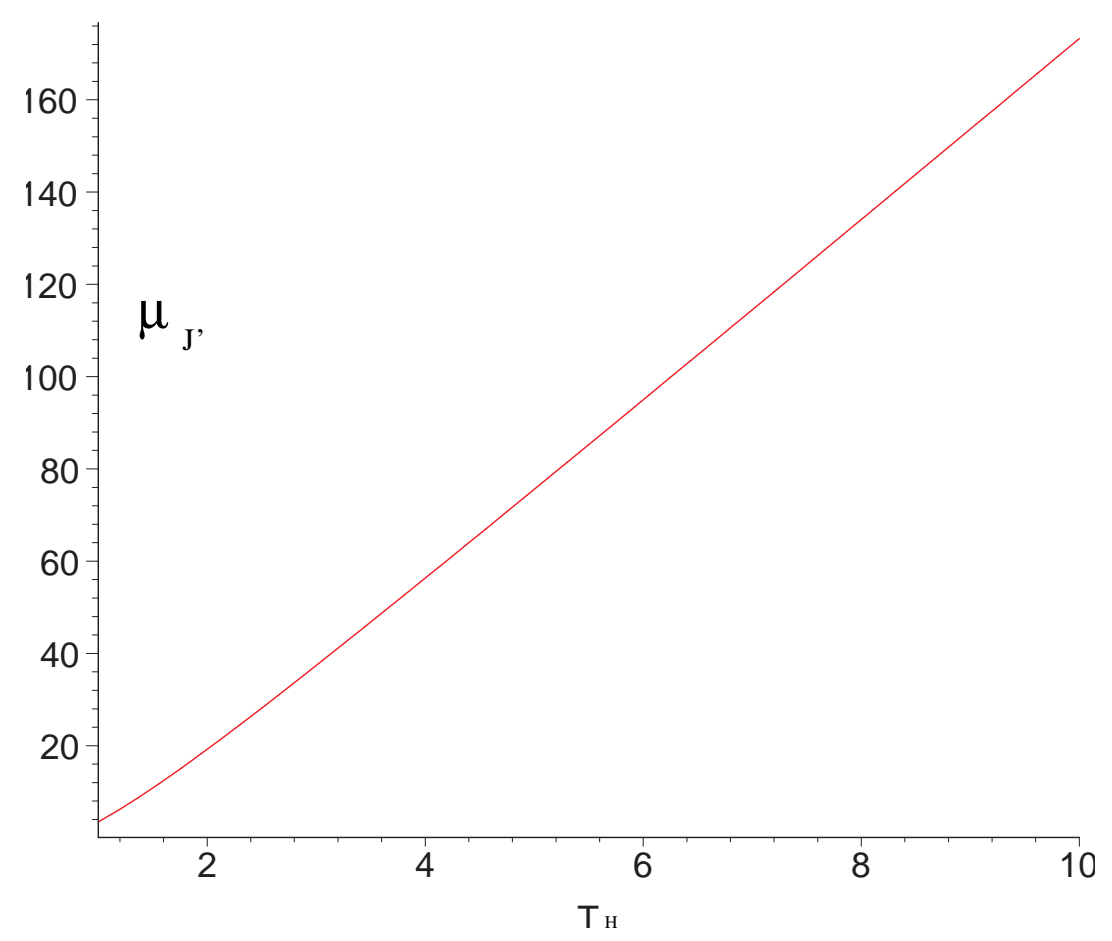

Figure 4: The chemical potential $\mu_{J^{\prime}}$ as a function of the Hagedorn temperature $T_{H}$ for strings in the maximally supersymmetric plane wave with null RR five form field strength.

We will use $b^{\prime}=b / \sqrt{\lambda}$, or correspondingly introduce the chemical potential conjugate to $J^{\prime}=J / \sqrt{\lambda}$. Note that the latter quantity remains finite in the BMN limit. Thus, the equation for the Hagedorn temperature as a function of the chemical potential in gauge theory quantities takes the final form:

$$
\frac{1}{16}\left(\frac{1}{T_{H}}+\mu_{J^{\prime}}\right)=\sum_{p=1}^{\infty} \frac{1}{p}\left(1-(-1)^{p}\right) K_{1}\left(\frac{2 p}{T_{H}}\right) .
$$

The dependence of the chemical potential on the Hagedorn temperature is presented in Fig. 4. Notice that for high Hagedorn temperatures the relation between the chemical potential and temperature simplifies to

$$
\mu_{J^{\prime}} \approx 2 \pi^{2} T_{H} .
$$




\section{Hagedorn vs. Hawking-Page transition}

The main motivation of this paper is to compare the Hagedorn behavior of strings in the maximally supersymmetric plane wave background, and the phase transition of the large charge black holes in the global $A d S_{5}$ background. To reiterate, the hope is that these large charge black holes are holographically dual to the large R-charge sector of the $\mathcal{N}=4 \mathrm{SYM}$ at finite temperature, which in turn can be described by an exactly soluble string model. The idea is then to compare the regime of the phase transition of black holes and the Hagedorn transition of strings, and thus 'unify' the two pictures of the confinement/deconfinement phase transition in $\mathcal{N}=4 \mathrm{SYM}$ on $\mathbb{R} \times S^{3}$ : a Hagedorn behavior at $\lambda \ll 1$, as discussed in [15], and the Hawking-Page black hole transition at $\lambda \gg 1$, as discussed in [13]. In the rest of this section we explain that the regime of the criticality of large charge black holes appear to be vastly different from the regime of the Hagedorn behavior of strings in PP wave background.

In section 2 we studied the thermodynamics of large charge black holes in global $A d S_{5}$ geometry. We found that these nonextremal geometries have a phase transition which is, in a sense, a generalization of the Hawking-Page phase transition [14]. It was argued in [13] that the HP phase transition realizes the strong coupling gravitational dual to the confinement/deconfinement phase transition in $\mathcal{N}=4 S U(N)$ supersymmetric Yang-Mills theory on $\mathbb{R} \times S^{3}$ background. There is an important difference between the phase transition for the charged black holes and the HP one discussed in [13]. For the HP transition one has two geometries, and compares the difference of their free energies (or Euclidean gravitational action). In the limit of vanishing nonextremality, one of the geometries does not have a horizon, but is simply a global Euclidean $A d S_{5}$ with the appropriately periodically identified time direction. In study the phase transition, this geometry is then used as a reference for the subtraction ("regularization" in the language of [6] ) of the Euclidean black hole gravitational action. As we explained above, in the case of the charged $\mathrm{BH}$ this subtraction procedure leads to an infinite expression for the ADM mass of the BH. Rather, the appropriate generalization would seem to be the subtraction of the "charged" global $A d S$ geometry, which however does not have a horizon. Such a nonsingular geometry does not exist since it requires to take the limit $\mu \rightarrow 0$ with $q$ - fixed and this is a violation of the 'BPS bound'. This situation motivated the use of the regularization procedure, which does not require a reference

background. Luckily, this can be implemented with a straightforward application of 
the holographic renormalization ideas of [6]. The obvious drawback for the absence of the reference background is that from the gravity perspective, the phase transition is defined in a rather ad hoc manner, i.e., as the vanishing of the generalized free energy, (36). Nonetheless, this definition of the phase transition is well motivated from the perspective of the holographically dual gauge theory. We found, compare (37)-(39), that a large value of charge for the black hole at the critical (phase transition) temperature implies a finite nonzero value of the chemical potential. Also, large charge at criticality implies large critical temperature:

$$
\begin{aligned}
\left.T_{\text {critical }}\right|_{J \rightarrow \infty} & \propto J^{1 / 2}, \\
\left.\mu_{J}\left(T_{\text {critical }}\right)\right|_{J \rightarrow \infty} & \rightarrow \frac{N^{2}}{2} .
\end{aligned}
$$

In section 3 we reviewed, that quite opposite to the regime of the critically of large charge black holes (52), the Hagedorn regime of strings in PP wave background for high Hagedorn temperature requires large values of the chemical potential, (51).

\section{Conclusion}

We attempted to provide a more precise relation between the Hagedorn description and the Hawking-Page like phase transition for the confinement/deconfinement transition in four dimensional $\mathcal{N}=4 S U(N)$ theory on $S^{3}$, by introducing a chemical potential conjugate to the large $R$-charge. The hope was that though the exact quantization of strings in $A d S_{5} \times S^{5}$ is not known, for large R-charge the essential physics could be captured by a string dual to this large R-charge sector, which is exactly soluble.

Unfortunately, we found that this is not so. It is plausible that rephrasing this confinement/deconfinement phase transition in the language of the Hagedorn transition at strong coupling would require the more detailed understanding of strings in $A d S_{5} \times S^{5}$.

\section{Acknowledgments}

We are grateful to Finn Larsen, Jim Liu, Eliezer Rabinovici and Cobi Sonnenschein for useful discussions. LAPZ is especially thankful to D. Vaman for collaboration in relevant topics. This work is supported in part by the U.S. Department of Energy. 


\section{References}

[1] J. M. Maldacena, "The large $N$ limit of superconformal field theories and supergravity," Adv. Theor. Math. Phys. 2, 231 (1998) [Int. J. Theor. Phys. 38, 1113 (1999)] [arXiv:hep-th/9711200].

[2] O. Aharony, S. S. Gubser, J. M. Maldacena, H. Ooguri and Y. Oz, "Large N field theories, string theory and gravity," Phys. Rept. 323, 183 (2000) [arXiv:hepth/9905111].

[3] D. Berenstein, J. M. Maldacena and H. Nastase, "Strings in flat space and pp waves from N = 4 super Yang Mills," JHEP 0204, 013 (2002) [arXiv:hep-th/0202021].

[4] K. Behrndt, M. Cvetic and W. A. Sabra, "Non-extreme black holes of five dimensional N = 2 AdS supergravity," Nucl. Phys. B 553, 317 (1999) [arXiv:hepth/9810227].

[5] M. Cvetic and S. S. Gubser, "Phases of R-charged black holes, spinning branes and strongly coupled gauge theories," JHEP 9904, 024 (1999) [arXiv:hep-th/9902195].

[6] V. Balasubramanian and P. Kraus, "A stress tensor for anti-de Sitter gravity," Commun. Math. Phys. 208, 413 (1999) [arXiv:hep-th/9902121].

[7] G. T. Horowitz and R. C. Myers, "The AdS/CFT correspondence and a new positive energy conjecture for general relativity," Phys. Rev. D 59, 026005 (1999) [arXiv:hep-th/9808079].

[8] A. Buchel and J. T. Liu, "Thermodynamics of the $\mathrm{N}=2^{*}$ flow," arXiv:hepth/0305064.

[9] A. Chamblin, R. Emparan, C. V. Johnson and R. C. Myers, "Holography, thermodynamics and fluctuations of charged AdS black holes," Phys. Rev. D 60, 104026 (1999) [arXiv:hep-th/9904197].

[10] A. Chamblin, R. Emparan, C. V. Johnson and R. C. Myers, "Charged AdS black holes and catastrophic holography," Phys. Rev. D 60, 064018 (1999) [arXiv:hepth/9902170]. 
[11] S. S. Gubser, A. A. Tseytlin and M. S. Volkov, "Non-Abelian 4-d black holes, wrapped 5-branes, and their dual descriptions," JHEP 0109, 017 (2001) [arXiv:hep-th/0108205].

[12] O. Aharony and A. Buchel, work in progress.

[13] E. Witten, "Anti-de Sitter space, thermal phase transition, and confinement in gauge theories," Adv. Theor. Math. Phys. 2, 505 (1998) [arXiv:hep-th/9803131].

[14] S. W. Hawking and D. N. Page, "Thermodynamics Of Black Holes In Anti-De Sitter Space," Commun. Math. Phys. 87,

[15] B. Sundborg, "The Hagedorn transition, deconfinement and N = 4 SYM theory," Nucl. Phys. B 573, 349 (2000) [arXiv:hep-th/9908001].

[16] I. Bena, J. Polchinski and R. Roiban, "Hidden symmetries of the $\operatorname{AdS}(5) \times \mathrm{S}^{* *} 5$ superstring," arXiv:hep-th/0305116.

[17] L. A. Pando Zayas and D. Vaman, "Strings in RR plane wave background at finite temperature," arXiv:hep-th/0208066.

[18] B. R. Greene, K. Schalm and G. Shiu, "On the Hagedorn behaviour of pp-wave strings and N = 4 SYM theory at finite R-charge density," Nucl. Phys. B 652, 105 (2003) [arXiv:hep-th/0208163].

[19] R. C. Brower, D. A. Lowe and C. I. Tan, "Hagedorn transition for strings on pp-waves and tori with chemical potentials," Nucl. Phys. B 652, 127 (2003) [arXiv:hep-th/0211201].

[20] G. Grignani, M. Orselli, G. W. Semenoff and D. Trancanelli, "The superstring Hagedorn temperature in a pp-wave background," arXiv:hep-th/0301186.

[21] Y. Sugawara, "Thermal amplitudes in DLCQ superstrings on pp-waves," Nucl. Phys. B 650, 75 (2003) [arXiv:hep-th/0209145]. Y. Sugawara, "Thermal partition function of superstring on compactified pp-wave," arXiv:hep-th/0301035.

[22] R. R. Metsaev, "Type IIB Green-Schwarz superstring in plane wave RamondRamond background," Nucl. Phys. B 625, 70 (2002) [arXiv:hep-th/0112044].

R. R. Metsaev and A. A. Tseytlin, "Exactly solvable model of superstring in plane 
wave Ramond-Ramond background," Phys. Rev. D 65, 126004 (2002) [arXiv:hepth/0202109].

[23] A. M. Polyakov, "Gauge fields and space-time," Int. J. Mod. Phys. A 17S1, 119 (2002) [arXiv:hep-th/0110196].

[24] D. Berenstein and H. Nastase, "On lightcone string field theory from super YangMills and holography," arXiv:hep-th/0205048. 